\title{
An Application of Eulerian Graph to PI on $M_{n}(C)$
}

\author{
Songfa You, Hongyan Zhao, Yijun Feng, Ming Cao \\ School of Mathematics and Computer Science, Hubei University, Wuhan, China \\ Email: yousongfa@163.com
}

Received April 25, 2012; revised May 31, 2012; accepted June 6, 2012

\begin{abstract}
We obtain a new class of polynomial identities on the ring of $n \times n$ matrices over any commutative ring with 1 by using the Swan's graph theoretic method [1] in the proof of Amitsur-Levitzki theorem. Let $\Gamma$ be an Eulerian graph with $k$ vertices and $d$ edges. Further let $n \geq 1$ be an integer and assume that $d \geq 2 \mathrm{kn}$. We proof that

$\sum_{\pi \in \Pi(\Gamma)} \operatorname{sgn}(\pi) x_{\pi(1)} x_{\pi(2)} \cdots x_{\pi(d)}=0$ is an PI on $M_{n}(C)$. Standard and Chang [2] Giambruno-Sehgal [3] polynomial identities are the special examples of our conclusions.
\end{abstract}

Keywords: Eulerian Graph; Eulerian Path; Admissible; Polynomial Identity

\section{Introduction}

Let $\Gamma$ be a finite directed graph with multiple edges allowed, and let $V(\Gamma)=\{1, \cdots, k\}$ denote the vertex set of $\Gamma$ and $E(\Gamma)=\left\{e_{1}, \cdots, e_{d}\right\}$ the edges set of $\Gamma$. Let $\sigma$ and $\tau$ be the functions from $E(\Gamma)$ to $V(\Gamma)$ defined by $\left(\sigma\left(e_{s}\right), \tau\left(e_{s}\right)\right)=(i, j)$ where $e_{s}$ is an edge from vertex $i$ to vertex $j$. For a vertex $i \in V(\Gamma)$ we put

$$
\phi_{+}(i)=\left|\left\{e_{s} \mid \sigma\left(e_{s}\right)=i\right\}\right|, \phi_{-}(i)=\left|\left\{e_{s} \mid \tau\left(e_{s}\right)=i\right\}\right|
$$

and

$$
\gamma(i)=\max \left\{\phi_{+}(i), \phi_{-}(i)\right\}
$$

We say that $e_{\pi(1)} e_{\pi(2)} \cdots e_{\pi(N)}$ is an Eulerian path of $\Gamma$ if $\pi$ is an element of $\operatorname{Sym}(d)$ (the symmetric group acting on the set $\{1, \cdots, d\}$ and $\tau\left(e_{\pi(i)}\right)=\sigma\left(e_{\pi(i+1)}\right)$ for $i=1, \cdots, d-1$.

It is well known that a connected graph $\Gamma$ has an Eulerin path starting at vertex $p$ and ending at vertex $q$ if and only if one of the following two conditions applies:

1) $p=q$ and $\phi_{+}(i)=\phi_{-}(i)$ for each $i=1, \cdots, k$;

2) $p \neq q$ and $\phi_{+}(p)=\phi_{-}(q)+1, \quad \phi_{-}(q)=\phi_{+}(q)+1$ and $\phi_{+}(i)=\phi_{-}(i)$ for each $i \in\{1, \cdots, k\} \backslash\{p, q\}$.

A directed connected graph $\Gamma_{p, q}$ with fixed vertices $p$ and $q$ is called Eulerian if either condition 1) or 2) is satisfied. We note that if $\Gamma_{p, q}$ is an Eulerian graph of type $(b)$, then the vertices $p, q$ are uniquely determined, but in the other case we may choose any vertex $p=q$. For an Eulerian graph $\Gamma_{p, q}$ denote by
$\Pi\left(\Gamma_{p, q}\right)=\left\{\pi \in \operatorname{Sym}(d) \mid e_{\pi(1)} \cdots e_{\pi(d)}\right.$ is an Eulerian path of $\Gamma_{p, q}$ starting at vertex $p$ and ending at vertex $\left.q\right\}$.

\section{Main Results}

Let $\Gamma$ be an Eulerian graph with $d$ edges $e_{1}, e_{2}, \cdots, e_{d}$ and distinguished points $p$ and $q$. the polynomial $f_{\Gamma}(X)$ associated with $\Gamma$ is defined as follows:

$$
f_{\Gamma}(X)=\sum_{\pi \in \Pi(\Gamma)} \operatorname{sgn}(\pi) x_{\pi(1)} X_{\pi(2)} \cdots x_{\pi(d)}
$$

Thus $f_{\Gamma}(X)$ is a multilinear polynomial in the set $X=\left\{x_{1}, \cdots, x_{d}\right\}$ of non-commuting indeterminates.

Let $n \geq 1$ be an integer, $C$ a commutative ring with 1 and $T: X \rightarrow\left\{E_{u v} \mid 1 \leq u, v \leq n\right\} \quad$ a set map where the $E_{u v}$ 's are the standard matrix units over $C$. It is clear that $T$ can be viewed as a substitution. we shall define a directed graph $\bar{\Gamma}_{T}$ induced from $\Gamma$ by $T$. First consider the directed graph on the vertex set $V \times\{1,2, \cdots, n\}$ with edge set $\bar{e}_{1}, \cdots, \bar{e}_{d}$ where $\sigma\left(\bar{e}_{r}\right)=\left(\sigma\left(e_{r}\right), u\right)$, $\tau\left(\bar{e}_{r}\right)=\left(\tau\left(e_{r}\right), v\right)$ and $x_{r}^{T}=E_{u v}$. Now we define $\bar{\Gamma}_{S}$ by restricting the vertex set to $\bigcup_{r=1}^{d}\left\{\sigma\left(\bar{e}_{r}\right), \tau\left(\bar{e}_{r}\right)\right\}$. We note that the graph so obtained need by no means be connected let alone Eulerian. If it is Eulerian however, by construction $\bar{\Gamma}_{T}$ has at most $\sum_{i=1}^{k} \min \{n, \gamma(i)\}$ vertices, where $\gamma(i)=\max \left\{\phi_{+}(i), \phi_{-}(i)\right\}$, i.e., $\gamma(i)=\phi_{+}(i)=\phi_{-}(i)$ for all $i \in V \backslash\{p, q\}$ and $\gamma(p)=\phi_{+}(p), \gamma(q)=\phi_{-}(q)$. Those elements of $\Pi(\Gamma)$ which do lift to an Eulerian path of $\bar{\Gamma}_{T}$ will be called admissible (with respect to $T$ ). It is clear that $\pi \in \Pi(\Gamma)$ is admissible if and only if $\bar{e}_{\pi(1)} \cdots \bar{e}_{\pi(d)}$ is an Eulerian 
path of $\bar{\Gamma}_{T}$. For the remainder of this section, we introduce Swan's theorem and our main results.

Swan [1]. Let $\Gamma$ be an Eulerian graph with $d$ edges and $k$ vertices satisfying $d \geq 2 k$. Then $\Pi(\Gamma)$ has the same number of odd and even permutations (with respect to the fixed order)

Theorem 1. Let $\Gamma$ be an Eulerian graph with vertex set $V=\{1,2, \cdots, k\}$ and $d$ edges. Further let $n \geq 1$ be an integer such that

$$
d \geq 2\left(\sum_{i=1}^{i} \min \{n, \gamma(i)\}\right)
$$

Then $f_{\Gamma}(X)=0$ is a polynomial identity on the ring $M_{n}(C)$ of $n \times n$ matrices over a commutative ring $C$ with 1

Corollary 2. Let $\Gamma$ be an Eulerian graph with $k$ vertices and $d$ edges. Further let $n \geq 1$ be an integer and assume that $d \geq 2 \mathrm{kn}$. Then $f_{\Gamma}(X)=0$ is a polynomial identity on $M_{n}(C)$.

\section{Proof of Theorem 1}

Since $f_{\Gamma}(X)$ is multilinear, it suffices to show that $f_{\Gamma}\left(X^{T}\right)=0$ for any substitution $T$ of $n \times n$ matrix units over $C$. Fix such an $T$ and put $x_{r}^{T}=E_{u(r) v(r)}$, $1 \leq r \leq d$. Then

$$
f_{\Gamma}\left(X^{T}\right)=\sum_{\pi \in \Pi(\Gamma)} \operatorname{sgn}(\pi) E_{u(\pi(1)) v(\pi(1))} \cdots E_{u(\pi(d)) v(\pi(d))}
$$

Now consider $\bar{\Gamma}_{T}$. Clearly, and summand in $\left(^{*}\right)$ vanishes unless, for the given $\pi \in \Pi(\Gamma)$,

$$
v(\pi(r))=u(\pi(r+1))
$$

for all $1 \leq r \leq N-1$, i.e., if $\pi$ is admissible. If so, on multiplying the matrix units, we obtain

$$
\operatorname{sgn}(\pi) E_{u(\pi(1)) v(\pi(d))} .
$$

It follows that

$$
f_{\Gamma}\left(X^{T}\right)=\sum_{u, v}\left(\sum \operatorname{sgn}(\pi)\right) E_{u v},
$$

where the inner sum is taken over all admissible permutations with $u(\pi(1))=u$ and $v(\pi(d))=v$. If no such admissible $\pi$ exists, the inner sum is 0 by definition. We want to prove that this inner sum is 0 anyway. It is readily seen that for any choice of $u$ and $b, a$ sum and $\operatorname{sgn}(\pi)$ in the inner sum arises precisely of $\pi$ lifts to an Eulerian path of $\bar{\Gamma}_{T}$ from $(p,(u)$ to $(q, v)$. Thus, on applying Swan's theorem to $\bar{\Gamma}_{T}$ with $\left|E\left(\bar{\Gamma}_{T}\right)\right|=d$ and $\left|V\left(\bar{\Gamma}_{T}\right)\right| \leq \sum_{i=1}^{k} \min \{n, \gamma(i)\}$, we find that the number of even and odd admissible permutations $\pi$ with $u(\pi(1))=u$ and $v(\pi(d))=v \quad$ coincide whence the inner sum is 0 for any choice of $u$ and $v$. This completes the proof.

\section{Applications}

1) Let $\Gamma$ be the Eulerian graph on one vertex with $d$ loops. Then $\Pi(\Gamma)=\operatorname{Sym}(d)$ and

$$
f_{\Gamma}(X)=\sum_{\pi \in \operatorname{Sym}(d)} \operatorname{sgn}(\pi) x_{\pi(1)} \cdots x_{\pi(d)}
$$

the standard polynomial [2] in $d$ indeterminates.

More generally, let $\Gamma$ be the Eulerian graph on $k$ vertices with distinguished points $p=q=1$ and the number $\alpha(i, j)$ of edges from vertex $i$ to $j$ :

$$
\alpha(i, j)= \begin{cases}m & \text { if } j=i+1 \text { and } 1 \leq i \leq k-1 \\ m & \text { if } i=k \text { and } j=1 \\ 0 & \text { otherwise }\end{cases}
$$

Now clearly $d=k m$ and

$$
\Pi(\Gamma)=\operatorname{sym}(m) \times \cdots \times \operatorname{sym}(m),
$$

$k$ times. On putting $\pi=\pi_{1} \times \cdots \times \pi_{k}$ and labelling the indeterminates, corresponding to the edges from $i$ to $i+1$ by $x_{1}^{(i)}, \cdots, x_{m}^{(i)}$, from the corollary 2 it follows that

$$
f_{\Gamma}(X)=\sum_{\pi \in \Pi(\Gamma)} \operatorname{sgn}(\pi)\left(\prod_{r=1}^{m} x_{\pi_{1}(r)}^{(1)} \cdots x_{\pi_{k}(r)}^{(k)}\right)=0
$$

is $a$ polynomial identity on $M_{n}(C)$ [3] if $k m=d \geq 2 \mathrm{kn}$, i.e., if $m \geq 2 n$.

2) For $\pi \in \Pi(\Gamma)$ we define a sequence $g(1), g(2), \cdots$, $g(d+1)$ of staircase steps, and the staircase height $g(\pi)=\max \{g(1), g(2), \cdots, g(d+1)\}$. We will construct a substitution $T$, such that $\pi$ lifts to the unique convering directed path of $\bar{\Gamma}_{S}$ (i.e., $\left.\Pi\left(\bar{\Gamma}_{S}\right)=\{\pi\}\right)$. First define a function $\pi^{*}:\{1,2, \cdots, d, d+1\} \rightarrow A$ by

$$
\begin{gathered}
\pi^{*}(1)=\sigma\left(e_{\pi(1)}\right) ; \pi^{*}(r)=\sigma\left(e_{\pi(r)}\right)=\tau\left(e_{\pi(r-1)}\right), \\
2 \leq r \leq d, \pi^{*}(d+1)=\tau\left(e_{\pi(d)}\right) .
\end{gathered}
$$

Next we define by recursion the sequence of pair $\left(g(r), w_{r}\right), \quad 1 \leq r \leq d+1$, where $g(r)$ is a natural number and $w_{r}$ is a subset of $\{1,2, \cdots, d, d+1\}$. We put $g(1)=1$ and $w_{1}=\phi$. Having $\left(g(1), w_{1}\right), \cdots,\left(g(r), w_{r}\right)$ in hand $(1 \leq r \leq d)$. There are three cases to consider:

a) $\pi^{*}(r+1) \neq \pi^{*}(t), \forall 1 \leq t \leq r$,

b) $\pi^{*}(r+1)=\pi^{*}(t), \pi^{*}(r+1) \neq \pi^{*}(s), \quad \forall t+1 \leq s \leq r$, $t \in w_{r}$

c) $\pi^{*}(r+1)=\pi^{*}(t), \pi^{*}(r+1) \neq \pi^{*}(s), \quad \forall t+1 \leq s \leq r$, $t \notin w_{r}$.

We now put 


$$
g(r+1)= \begin{cases}1 & \text { in case (1) } \\ g(t)+1 & \text { in case (2) } \\ g(t) & \text { in case (3) }\end{cases}
$$

and

$$
w_{r+1}= \begin{cases}w_{r} & \text { in case (1) and (2) } \\ w_{r} \cup\{t, t+1, \cdots, r, r+1\} & \text { in case (3). }\end{cases}
$$

Let $n \geq g(r)$ for all $1 \leq r \leq d+1$, it is clear that $x_{r}^{T}=E_{g(r) g(r+1)}(1 \leq r \leq d)$ gives $a$ substitution of $n \times n$ matrix units over $C$. Now

$$
\begin{aligned}
& \left(\pi^{*}(1), g(1)\right) \stackrel{\bar{e}_{\pi(1)}}{\longrightarrow}\left(\pi^{*}(2), g(2)\right) \rightarrow \cdots \rightarrow \\
& \left(\pi^{*}(d), g(d)\right) \stackrel{\bar{e}_{\pi(d)}}{\longrightarrow}\left(\pi^{*}(d+1), g(d+1)\right)
\end{aligned}
$$

is the unique covering directed path of $\bar{\Gamma}_{S}$ from $\left(\pi^{*}(1), g(1)\right)$ to $\left(\pi^{*}(d+1), g(d+1)\right)$ [4-7]. Since the $(g(1), g(d+1))$ entry of the $n \times n$ matrix $f_{\Gamma}\left(X^{T}\right)$ is $\operatorname{sgn}(\pi)$, we have

Theorem 3. Let $\Gamma$ be an Eulerian graph and $\pi \in \Pi(\Gamma)$. If $n \geq g(\pi)$, then $f_{\Gamma}(X)=0$ is not $a$ polynomial identity on the ring $M_{n}(C)$ of $n \times n$ matrices over a commutative ring $C$ with 1 .

Remark. It is an obvious consequence of the above theorem that if $n \geq \min \{g(\pi) \mid \pi \in \Pi(\Gamma)\}$ is not the least integer $n \geq 1$ for which $f_{\Gamma}(X)=0$ is not $a$ polynomial identity on $M_{n}(C)$.

We note that, in general $\min \{g(\pi) \mid \pi \in \Pi(\Gamma)\}$ is not the least integer $n \geq 1$ for which $f_{\Gamma}(X)=0$ is not $a$ polynomial identity on $M_{n}(C)$.

Let $\Gamma$ be the Eulerian graph on one vertex $d$ loops. It is easily see that

$$
\begin{gathered}
g(1)=g(2)=1, g(3)=g(4)=2, \cdots, \\
g(2 s+1)=g(2 s+2)=s+1, \cdots .
\end{gathered}
$$

Thus $g(\pi)=[d / 2]+1$ for all $\pi \in \operatorname{sym}(d)$ and the minimality assertion of the Amitsur-Levitzki theorem follows; the main part is an immediate consequence of the corollary.

Let $\Gamma$ be the Eulerian graph on $k$ vertices with distinguished points $p=q=1$ and the number $\alpha(i, j)$ of edges from vertex $i$ to $j$ :

$$
\alpha(i, j)= \begin{cases}m & \text { if } j=i+1 \text { and } 1 \leq i \leq k-1 \\ m & \text { if } i=k \text { and } j=1 \\ 0 & \text { otherwise }\end{cases}
$$

Analogously, for any $\pi \in \Pi(\Gamma)$ we have

$$
\begin{gathered}
g(1)=g(2)=\cdots=g(k+1)=1, \\
g(k+2)=g(k+3)=\cdots=g(2 k+2)=2, \\
\cdots g(s(k+1)+1)=g(s(k+1)+2)= \\
\cdots=g((s+1)(k+1))=s+1 .
\end{gathered}
$$

In consequence $g(\pi)=m-[(m-1) /(k+1)]$ for all $\pi \in \Pi(\Gamma)$.

For $k=2$ we get the double Capelli polynomial; it is known, however, that in this case $m-[(m-1) / 3]$ is not the smallest $n$ for which $f_{\Gamma}(X)=0$ is not $a$ polynomial identity on $M_{n}(C)$.

When $k=3$ we use $x, y$ and $z$ instead of the symbols $x^{(1)}, x^{(2)}$, and $x^{(3)}$ respectively to denote the indeterminates of the triple Capelli polynomial and continue to write $m$ for the number of edges from vertex $i$ to $i+1$. Thus the triple Capelli polynomial is

$$
\begin{aligned}
& C_{M}(X, Y, Z) \\
& =\sum_{\pi \in \Pi(\Gamma)} \operatorname{sgn}(\pi) x_{\pi_{1}(1)} y_{\pi_{2}(1)} Z_{\pi_{3}(1)} \cdots x_{\pi_{1}(m)} y_{\pi_{2}(m)} Z_{\pi_{3}(m)} .
\end{aligned}
$$

\section{REFERENCES}

[1] R. G. Swan, "An Application of Graph Theory to Algebra," Proceedings of the American Mathematical Society, Vol. 14, 1963, pp. 367-373.

[2] Q. Chang, "Some Consequences of the Standard Polynomial," Proceedings of the American Mathematical Society, Vol. 104, 1988, pp. 707-710. doi:10.1090/S0002-9939-1988-0964846-8

[3] A. Giambruno and S. K. Sehgal, "On a Polynomial Identity for $n \times n$ Matrices," Journal of Algebra, Vol. 126, No. 2, 1989, pp. 451-453. doi:10.1016/0021-8693(89)90312-8

[4] S. F. You, Y. M. Zheng and D. G. Hu, "Eulerian Graph and Polynomial Identities on Matrix Rings," Advances in Math, Vol. 32, 2003, pp. 425-428.

[5] S. F. You, "The Primitivity of Extended Centroid Extension on Prime GPI-Rings," Advances in Math, Vol. 29, 2000, pp. 331-336.

[6] S. F. You, "The Essential (One-Sided) Ideal of Semiprime PI-Rings," Acta Mathematica Sinica, Vol. 44, 2001, pp. 747-752.

[7] S. F. You, M. Cao and Y. J. Feng, "Semiautomata and Near Rings," Quantitative Logic and Soft Computing, Vol. 5, 2012, pp. 428-431. doi:10.1142/97898144015310058 